Pacific Journal of Mathematic 


\title{
GLOBAL ANALYSIS AND PERIODIC SOLUTIONS OF SECOND ORDER SYSTEMS OF NONLINEAR DIFFERENTIAL EQUATIONS
}

\section{DAVID Westreich}

\begin{abstract}
We establish the existence of global closed connected sets of solutions of nonlinear operator equations with linearized part a polynomial in $\lambda$, bifurcating from characteristic values of odd multiplicity. These results are then applied to finding large periodic solutions of systems of second order nonlinear differential equations.
\end{abstract}

Introduction. Using Leray-Schauder degree theory P. H. Rabinowitz [10] has shown the existence of global continua of solutions bifurcating from characteristic values of odd multiplicity of the linearized part of completely continuous nonlinear operator equations. By purely local bifurcation results, unrelated to those of Rabinowitz, the author [13] has extended M. S. Berger's [1] variational techniques to find small periodic solutions of systems of second order nonlinear differential equations. In this paper we combine the two methods and develop Rabinowitz's global analysis for nonlinear operators whose linearized part is a polynomial in $\lambda$ and apply these results to the existence of large periodic solutions of second order differential equations of the form

$$
\begin{aligned}
& u^{\prime \prime}+A_{1} v^{\prime}+B_{1} u+F_{1}\left(w, w^{\prime}, w^{\prime \prime}\right)=0 \\
& v^{\prime \prime}+A_{2} u^{\prime}+B_{2} v+F_{2}\left(w, w^{\prime}, w^{\prime \prime}\right)=0
\end{aligned}
$$

where $w=(u, v)$.

1. Global analysis. We consider the existence of continua of solutions of equations of the form

$$
x=\lambda L x+G(\lambda, x)
$$

where $L$ is a completely continuous linear map of a real Banach space $X$ into itself and $G(\lambda, x)$ is a completely continuous map [12, p. 9] of $R \times X \rightarrow X$ satisfying $G(\lambda, x)=o(\|x\|)$ for $x$ near zero, uniformly on bounded $\lambda$ intervals. A solution of Eq. (1.1) is a point $(\lambda, x) \in \boldsymbol{R} \times X$ satisfying Eq. (1.1) and will be called trivial if $x=0$. A $\lambda_{0} \in \boldsymbol{R}$ is said to be a bifurcation point if every neighborhood of $\left(\lambda_{0}, 0\right)$ contains nontrivial solutions of Eq. (1.1). The closure of the set of nontrivial solutions of (1.1) will be denoted $S$.

A $\lambda_{0} \in \boldsymbol{R}$ will be called a characteristic value of a linear operator $L$ if there exists a nonzero $x_{0} \in X$ such that $x_{0}=\lambda_{0} L x_{0}$. The set of 
characteristic values of $L$ will be denoted $r(L)$. The domain, range and null space of an operator $B$ will be denoted by $D(B), R(B)$ and $N(B)$ respectively. For convenience we will let $N_{k}(B)=N\left(B^{k}\right), N_{\infty}(B)=$ $\mathrm{U}_{i=1}^{\infty} N\left(B^{i}\right)$, and $R_{k}(B)=R\left(B^{k}\right)$. The smallest integer $k>0$ such that $N_{k}(B)=N_{\infty}(B)$ will be denoted $\alpha(B)$. The algebraic multiplicity, $M\left(\lambda_{0}, L\right)$, of $\lambda_{0} \in r(L)$ is $\operatorname{dim} N_{\infty}\left(I-\lambda_{0} L\right)$.

Suppose that $G$ is not globally defined. Then by employing an obvious modification of E. N. Dancer's work [4, Corollary 2] we can obtain the following extension of Rabinowitz's Theorem 1.12 in [10].

THEOREM 1.1. Let $P=\left\{\lambda_{1}, \cdots, \lambda_{p}\right\}$ be a set of consecutive characteristic values of $L, \lambda_{1}<\cdots<\lambda_{P}$, and suppose $\sum_{i=1}^{p} M\left(\lambda_{i}, L\right)$ is odd. If $\Omega$ is a bounded open set in $\boldsymbol{R} \times X$ containing $\left[\lambda_{1}, \lambda_{p}\right] \times\{0\}$ and $G(\lambda, x)$ is completely continuous on $\bar{\Omega}$ then $S$ possesses a maximal subcontinuum $C_{P}$ such that $\left(\lambda_{j}, 0\right) \in C_{P}$ for some $\lambda_{j} \in P$ and $C_{P}$ either

(i) meets $\partial \Omega$ or

(ii) meets $(\hat{\lambda}, 0) \in \Omega$, where $\hat{\lambda} \in(r(L)-P)$.

By a subcontinuum of $S$ we mean a subset of $S$ which is closed and connected in $\boldsymbol{R} \times X$. By $\partial \Omega$ we mean of course the boundary of $\Omega$.

With Theorem 1.1 we can obtain global results for $G(\lambda, x)$ globally defined but now $L$ is just a bounded linear map of $X$ into $X$ such that for all $\lambda \in \boldsymbol{R}, R(I-\lambda L)$ is closed and $N_{\infty}(I-\lambda L), N_{\infty}\left(I-\lambda L^{*}\right)<$ $\infty, L^{*}$ the conjugate of $L$. With these assumptions on $L$ we have the lemma

Lemma 1.2. If $k=\alpha(I-\lambda L)$ then $R_{k}(I-\lambda L)$ is closed, $X=$ $N_{k}(I-\lambda L) \oplus R_{k}(I-\lambda L)$ and $I-\lambda L$ is a one-one map of $R_{k}(I-\lambda L)$ onto $R_{k}(I-\lambda L)$ with bounded inverse.

\section{Proof. See [14].}

Lemma 1.2 implies that if $1 / \lambda$ is in the spectrum of $L$ then $\lambda \epsilon$ $r(L)$ and $r(L)$ is discrete.

Theorem 1.3. Let $L$ and $G$ be as described above and let $P=$ $\left\{\lambda_{1}, \cdots, \lambda_{p}\right\}$ be a set of consecutive characteristic values of $L, \lambda_{1}$ $<\cdots<\lambda_{p}$. Suppose $\sum_{i=1}^{p} M\left(\lambda_{i}, L\right)$ is odd. Then $S$ contains a maximal subcontinuum $C_{P}$ such that $\left(\lambda_{j}, 0\right) \in C_{P}$ for some $\lambda_{j} \in P$ and $C_{P}$ is either

(i) unbounded in $\boldsymbol{R} \times X$ or

(ii) meets $(\hat{\lambda}, 0)$, where $\hat{\lambda} \in(r(L)-P)$. 
Proof. The theorem will be proved by repeated applications of Theorem 1.1. For each positive integer $n$ define $I_{n}=\left(\lambda_{1}-n, \lambda_{p}+n\right)$ and $S_{n}=\{x \in X \mid\|x\|<n\}$. Let $\lambda_{1}, \cdots, \lambda_{p}, \cdots, \lambda_{s}$ be the characteristic values of $L$ contained in $\bar{I}_{n}$. Then by Lemma $1.3, X=N \oplus R$, where

$$
\begin{aligned}
& N=N_{k_{1}}\left(I-\lambda_{1} L\right) \oplus \cdots \oplus N_{k_{s}}\left(I-\lambda_{s} L\right) \\
& k_{i}=\alpha\left(I-\lambda_{i} L\right), \quad i=1, \cdots s
\end{aligned}
$$

and

$$
R=R\left(\left(I-\lambda_{1} L\right)^{k_{1}} \cdots\left(I-\lambda_{s} L\right)^{k_{s}}\right) .
$$

Thus $x \in X$ can be uniquely expressed as $x=u+v$ where $u \in N$ and $v \in R$. Moreover, for all $\lambda \in \boldsymbol{R}, I-\lambda L: N \rightarrow N, R \rightarrow R$ and $G=G_{N}+$ $G_{R}$ where $G_{N} \in N$ and $G_{R} \in R$. Thus our problem is equivalent to that of finding solutions $(\lambda, u+v) \in \boldsymbol{R} \times(N \oplus R)$ of the system of equations

$$
\begin{aligned}
& u-\lambda L u=G_{N}(\lambda, u+v) \\
& v-\lambda L v=G_{R}(\lambda, u+v) .
\end{aligned}
$$

Since $I-\lambda L$ has a bounded inverse on $R$ for all $\lambda \in \bar{I}_{n}$ and $(I-\lambda L)^{-1}$ is continuous in $\lambda$ for all $1 / \lambda$ in the resolvent of $L$ (as a mapping of $R \rightarrow R$ ) [5, p. 257], $(\lambda, u+v) \in \bar{\Omega}_{n}=\bar{I}_{n} \times S_{n}$ is a solution of (1.2), (1.3) if and only if $(\lambda, u+v)$ is a solution of the system

$$
\begin{gathered}
u=\lambda L u+G_{N}(\lambda, u+v) \\
v=(I-\lambda L)^{-1} G_{R}(\lambda, u+v) .
\end{gathered}
$$

As $(I-\lambda L)^{-1} G_{R}(\lambda, u+v)$ is a completely continuous map of $\bar{\Omega}_{n}$ into $R$ and $\operatorname{dim} N<\infty$, Eqs. (1.4), (1.5) satisfy the hypothesis of Theorem 1.1. Thus for some $1 \leqq j \leqq p$ and each integer $n, \bar{\Omega}_{n}$ contains a maximal closed connected subset $C_{n}$ of $S$ such that $\left(\lambda_{j}, 0\right) \in C_{n}$ and $C_{n}$ either meets the boundary of $\Omega_{n}$ or meets $(\hat{\lambda}, 0), \hat{\lambda} \in(r(L)-P)$. As the union of connected sets containing a common point is connected, an application of Zorn's lemma [8, p. 62] will show that $S$ contains a unique maximal subcontinuum $C_{P}$ meeting $\left(\lambda_{j}, 0\right)$. Thus $\mathrm{U}_{n=1}^{\infty} C_{n} \subset C_{P}$ and our theorem is proved.

REMARK. If $G$ is not globally defined but is instead completely continuous on the closure of an open bounded set $\Omega \subset \boldsymbol{R} \times X$, containing $\left[\lambda_{1}, \lambda_{p}\right] \times\{0\}$, and $L$ and $P$ are as in Theorem 1.3 , then by replacing the $\Omega_{n}$ by $\Omega$ we can readily prove that if $\sum_{i=1}^{p} M\left(\lambda_{i}, L\right)$ is odd, $S$ possesses a maximal subcontinuum $C_{P} \subset \bar{\Omega}$ such that $C_{P} \cap$ $(P \times\{0\}) \neq \varnothing$ and either (i) meets $\partial \Omega$ or (ii) meets $(\widehat{\lambda}, 0) \in \bar{\Omega}$, where 
$\hat{\lambda} \in(r(L)-P)$.

If we consider (1.4) and (1.5) and modify Rabinowitz's proof of Theorem 1.16 in [1], (as was done in [4]) for $C_{P}$ in a sufficiently large $\Omega_{n}$ we can readily verify

THEOREM 1.4. Suppose the hypotheses of Theorem 1.3 are satisfied and (i) does not occur. Then $C_{P}$ meets a point $(\hat{\lambda}, 0)$ such that $\hat{\lambda} \in$ $(r(L)-P)$ and $M(\hat{\lambda}, L)$ is odd.

REMARK. Similarly we could show that when a $G$ is not globally defined and (i) of the above remark does not obtain then $C_{P}$ meets a $(\hat{\lambda}, 0)$ and $M(\hat{\lambda}, L)$ is odd.

The existence of continua of nontrivial solutions can also be shown for completely continuous operator equations of the form

$$
x=\left(\lambda^{n} A_{n}+\cdots+\lambda A_{1}\right) x+G(\lambda, x)
$$

where $G(\lambda, x)$ is $o(\|x\|)$ for $x$ near zero, uniformly on bounded $\lambda$ intervals and $A_{1}, \cdots, A_{n}$ are completely continuous linear operators of a real Banach space $X$ into itself. We will call $\lambda_{0}$ a characteristic value of an operator polynomial $A(\lambda)=\lambda^{n} A_{n}+\cdots+\lambda A_{1}$ if there exists a nonzero vector $x_{0} \in X$ such that $A\left(\lambda_{0}\right) x_{0}=x_{0}$. The set of such characteristic values will be denoted $r(A)$.

The geometric multiplicity of $\lambda_{0} \in r(A)$ is $\operatorname{dim} N\left(I-A\left(\lambda_{0}\right)\right)$. To define its algebraic multiplicity we consider the associated matrix of operators $W+V: X^{n} \rightarrow X^{n}$, where

$$
W=\left[\begin{array}{cccccc}
0 & \cdot & \cdot & \cdot & 0 & A_{n} \\
\cdot & & & & \cdot & \cdot \\
\cdot & & & & \cdot & \cdot \\
\cdot & & & & \cdot & \cdot \\
0 & \cdot & \cdot & \cdot & 0 & A_{1}
\end{array}\right]
$$

and

$$
V=\left[\begin{array}{cccccc}
0 & 0 & . & . & \cdot & 0 \\
I & 0 & . & . & . & 0 \\
0 & I & . & . & . & 0 \\
\cdot & . & . & . & . & . \\
0 & . & . & . & I & 0
\end{array}\right]
$$

If $x=\left(x_{1}, \cdots, x_{n}\right), x_{1}, \cdots, x_{n} \in X$, then the operator equation $x=$ $\lambda(W+V) x$ is the system of equations 


$$
\begin{aligned}
x_{1} & =\lambda A_{n} x_{n} \\
x_{2} & =\lambda\left(A_{n-1} x_{n}+x_{1}\right) \\
& \vdots \\
x_{n} & =\lambda\left(A_{1} x_{n}+x_{n-1}\right) .
\end{aligned}
$$

Thus it is clear $\lambda_{0} \in r(A)$ if and only if $\lambda_{0} \in r(W+V)$. We therefore define the algebraic multiplicity $M\left(\lambda_{0}, A\right)$ of $\lambda_{0}$ to be the

$$
\operatorname{dim} N_{\infty}\left(I-\lambda_{0}(W+V)\right) \text {. }
$$

REMARK. Our definition is an analog of the one given for finite dimensional square matrices. The algebraic multiplicity of a characteristic value $\lambda_{0}$ of a matrix $A$ is the multiplicity of the eigenvalue $\beta_{0}=1 / \lambda_{0}$ as a root of the characteristic equation $\operatorname{det}|\beta I-A|=0[7, \mathrm{p}$. 104]. Correspondingly, the algebraic multiplicity of a characteristic value $\lambda_{0}$ of a matrix polynomial $A(\lambda)$ is the multiplicity of $\beta_{0}=1 / \lambda_{0}$ as a root of the equation

$$
\operatorname{det}\left|\beta^{n} I-A_{n}-\cdots-\beta^{n-1} A_{1}\right|=0 .
$$

Moreover, by the Jordan canonical form the algebraic multiplicity of a characteristic value $\lambda_{0}$ of a matrix $A$, is equal to $\operatorname{dim} N_{\infty}\left(I-\lambda_{0} A\right)$. Similarly, the algebraic multiplicity of a characteristic value $\lambda_{0}$ of $A(\lambda)$ is $\operatorname{dim} N_{\infty}\left(I-\lambda_{0}(W+V)\right)$. Indeed $\operatorname{dim} N_{\infty}\left(I-\lambda_{0}(W+V)\right)$ is equal to the multiplicity of $\beta_{0}=1 / \lambda_{0}$ as a root of the characteristic equation $\operatorname{det}|\beta I-W-V|=0$. But

$$
\operatorname{det}|\beta I-W-V|=\operatorname{det}\left|\beta^{n} I-A_{n}-\cdots-\beta^{n-1} A_{1}\right|
$$

since by elementary row operations we have

$$
\begin{aligned}
& \operatorname{det}|\beta I-W-V| \\
& =\left|\begin{array}{cccccc}
\beta I & 0 & \cdot & \cdot & \cdot & -A_{n} \\
-I & \beta I & \cdot & \cdot & \cdot & -A_{n-1} \\
\cdot & \cdot & \cdot & & & \cdot \\
\cdot & & \cdot & \cdot & & \cdot \\
\cdot & & & \cdot & \cdot & \cdot \\
0 & & & & -I & \beta I-A_{1}
\end{array}\right| \\
& =\left|\begin{array}{cccccc}
\beta I & 0 & \cdot & \cdot & \cdot & -A_{n} \\
0 & \beta I & & & -\beta^{-1} A_{n}-A_{n-1} \\
\cdot & \cdot & \cdot & \cdot & \cdot & \cdot \\
\cdot & \cdot & \cdot & \cdot \\
\cdot & & & & \cdot \\
0 & 0 & \cdot & \cdot & \cdot & \beta I-\beta^{-n+1} A^{n}-\cdots-A_{1}
\end{array}\right| \\
& =\operatorname{det}\left|\beta^{n} I-A_{n}-\cdots-\beta^{n-1} A_{1}\right| \text {. }
\end{aligned}
$$


The closure of the set of nontrivial solutions of Eq. (1.6) will be denoted by $S$. A $\lambda_{0} \in R$ will be a bifurcation point if every neighborhood of $\left(\lambda_{0}, 0\right)$ contains nontrivial solutions of Eq. (1.6). It is clear that if $\lambda_{0}$ is a bifurcation point of Eq. (1.6) then $\lambda_{0} \in r(A)$. To see this we note that by the Fredholm theory [11, Chaps. IV, V] if $\lambda_{0} \notin r(A)$ then $I-A(\lambda)$ is invertible for all $\lambda$ near $\lambda_{0}$ with uniformly bounded inverse. Writing (1.6) for $x \neq 0$ in the form

$$
x /\|x\|=(I-A(\lambda))^{-1} G(\lambda, x) /\|x\|
$$

we see $\lambda_{0}$ is not a bifurcation point of Eq. (1.6) as $G=o(\|x\|)$.

We can show

THEOREm 1.5. Let $P=\left\{\lambda_{1}, \cdots, \lambda_{p}\right\}$ be a set of consecutive characteristic values of $A(\lambda), \lambda_{1}<\cdots<\lambda_{p}$, and suppose $\sum_{i=1}^{p} M\left(\lambda_{i}, A\right)$ is odd. Then $S$ contains a maximal subcontinuum $C_{P}$ such that $\left(\lambda_{j}, 0\right) \in$ $C_{P}$ for some $\lambda_{j} \in P$ and $C_{P}$ is either

(i) unbounded in $\boldsymbol{R} \times X$ or

(ii) meets $(\hat{\lambda}, 0)$ where $\hat{\lambda} \in(r(A)-P)$.

Proof. Showing the existence of $C_{P}$ is equivalent to showing the existence of a maximal continuum of solutions $C_{P}^{\prime}$ of

$$
x=\lambda(W+V) x+\hat{G}(\lambda, x)
$$

in $X^{n}$, where

$$
\hat{G}(\lambda, x)=\hat{G}\left(\lambda, x_{1}, \cdots, x_{n}\right)=\left(0, \cdots, 0, G\left(\lambda, x_{n}\right)\right) .
$$

We will show Eq. (1.9) satisfies the hypotheses of Theorem 1.3. By (1.10) $\hat{G}(\lambda, x)$ is completely continuous and $\hat{G}(\lambda, x)=o(\|x\|)$ for $x$ near zero, uniformly on bounded $\lambda$ intervals. In addition, $\operatorname{dim} N_{\infty}(I-$ $\lambda(W+V)), \operatorname{dim} N_{\infty}\left(I-\lambda(W+V)^{*}\right)<\infty$ and $R(I-\lambda(W+V))$ is closed for all real $\lambda$. Indeed, one can readily verify $V^{n-1}=0$. Thus as $(W+V)^{m}=K_{m}+V^{m}$ for all positive integers $m$, where $K_{m}$ is completely continuous, it follows that $(V+W)^{n-1}$ is completely continuous. Moreover

$$
N_{\infty}(I-\lambda(W+V)) \subset N_{\infty}\left(I-\lambda^{n-1}(W+V)^{m-1}\right) .
$$

Thus by the Riesz-Schauder theorem [11, p. 183 and p. 219], $\operatorname{dim} N_{\infty}(I-$ $\lambda(W+V))$ is finite. Similarly as $\left((W+V)^{*}\right)^{n-1}$ is completely continuous, $\operatorname{dim} N_{\infty}\left(I-\lambda(W+V)^{*}\right)<\infty$. Lastly, as $(V+W)^{n-1}$ is completely continuous it readily follows that $R(I-\lambda(W+V))$ is closed [6, p. 705].

Hence Eq. (1.9) satisfies the hypothesis of Theorem 1.3 and the result follows. 
An argument analogous to that of the proof of Theorem 1.5 will show that if $G$ is not globally defined and $A(\lambda)$ and $P$ are as in Theorem 1.5 then

THEOREM 1.6. If $\Omega$ is a bounded open set in $\boldsymbol{R} \times X$ containing $\left[\lambda_{1}, \lambda_{p}\right] \times\{0\}$ and $G(\lambda, x)$ is completely continuous on $\bar{\Omega}$ and $\sum_{i=1}^{p} M\left(\lambda_{i}, A\right)$ $i s$ odd then $S$ possesses a maximal subcontinuum $C_{P} \subset \bar{\Omega}$ such that $\left(\lambda_{j}, 0\right) \in C_{P}$ for some $\lambda_{j} \in P$ and $C_{P}$ either

(i) meets $\partial \Omega$ or

(ii) meets $(\hat{\lambda}, 0) \in \Omega$, where $\hat{\lambda} \in(r(A)-P)$.

By arguing as in the proof of Theorem 1.5 and applying Theorem 1.4 or the remark following Theorem 1.4 we can verify

Theorem 1.7. Suppose the hypotheses of Theorem 1.5 (1.6) are satisfied and (i) does not occur. Then $C_{P}$ meets a point $(\hat{\lambda}, 0)$ such that $\hat{\lambda} \in(r(A)-P)$ and $M(\hat{\lambda}, A)$ is odd.

Theorem 1.6 can be used to show the existence of bifurcation points of operator equations which are not completely continuous. Suppose $A_{1}, \cdots, A_{n}$ are closed linear operators of $X$ into $X$ such that $\overline{\mathrm{\Pi}_{i=1}^{n} D\left(A_{i}\right)}=X$ and $\lambda_{0}$ is a characteristic value of $\lambda^{n} A_{n}+\cdots+\lambda A_{1}$. Let us further assume $G(\lambda, x)$ is a continuous map of a neighborhood of $\left(\lambda_{0}, 0\right) \in R \times X$ into $X$ satisfying

$$
\left\|G\left(\lambda, x_{1}\right)-G\left(\lambda, x_{2}\right)\right\| \leqq h\left(x_{1}, x_{2}\right)\left\|x_{1}-x_{2}\right\|
$$

for $\left(\lambda, x_{1}\right),\left(\lambda, x_{2}\right)$ near $\left(\lambda_{0}, 0\right)$ and where $h(a, b)$ is a function indepenent of $\lambda$ tending to zero as both $a$ and $b$ tend to zero. If we define $W$ and $V$ by (1.7) and (1.8) and let $S$ be the closure of the set of nontrivial solutions of

$$
x=\left(\lambda^{n} A_{n}+\cdots+\lambda A_{1}\right) x+G(\lambda, x)
$$

we can prove

THEOREM 1.8. If $I-\lambda_{0}(W+V)$ is a closed Fredholm operator of index zero [4] and $M\left(\lambda_{0}, A\right)$ is odd then $\lambda_{0}$ is a bifurcation point of $E q$. (1.12) and $S$ contains a maximal subcontinuum meeting $\left(\lambda_{0}, 0\right)$.

Proof. As in the proof of Theorem 1.5 it suffices to show $\lambda_{0}$ is a bifurcation point of

$$
x=\lambda(W+V) x+\hat{G}(\lambda, x)
$$

in $X^{n}$, where $\hat{G}$ is defined by (1.10). By hypothesis one can readily 
verify that $X^{n}=N_{p}\left(I-\lambda_{0}(W+V)\right) \oplus R_{p}\left(I-\lambda_{0}(W+V)\right)$ where $p=$ $\alpha\left(I-\lambda_{0}(W+V)\right)$ [14]. Thus for $x \in X^{n}, x=u+v$ where $u \in N_{p}(I-$ $\left.\lambda_{0}(W+V)\right)$ and $v \in R_{p}\left(I-\lambda_{0}(W+V)\right)$ and $\hat{G}=G_{N}+G_{R}$ where $G_{N} \in$ $N_{p}\left(I-\lambda_{0}(W+V)\right)$ and $G_{R} \in R_{p}\left(I-\lambda_{0}(W+V)\right)$. Moreover $I-\lambda(W+V)$ is invertible on $R_{p}\left(I-\lambda_{0}(W+V)\right)$ for all $\lambda$ near $\lambda_{0}$. Thus finding solutions of Eq. (1.12) is equivalent to finding solutions of the system

$$
\begin{aligned}
& u=\lambda(W+V) u+G_{N}(\lambda, u+v) \\
& v=(I-\lambda(W+V))^{-1} G_{R}(\lambda, u+v) .
\end{aligned}
$$

An application of the contraction mapping principle [5, p. 260] to Eq. (1.14) shows the existence of a uniquely determined continuous function $v(\lambda, u)$ such that $v(\lambda, u) \equiv(I-\lambda(W+V))^{-1} G_{R}(\lambda, u+v(\lambda, u))$. Hence our problem is reduced to that of solving the equation

$$
u=\lambda(W+V) u+G_{N}(\lambda, u+v(\lambda, u)) .
$$

As the equation satisfies the hypotheses of Theorem 1.6 there is a unique maximal subcontinuum of meeting $\left(\lambda_{0}, 0\right)$ and the result follows.

2. Second order systems. Let us consider autonomous systems of ordinary differential equations of the form

$$
w^{\prime \prime}+A_{1} w^{\prime}+A_{2} w+F\left(w, w^{\prime}, w^{\prime \prime}\right)=0
$$

where $w$ denotes the $n$-vector $\left(w_{1}(t), \cdots, w_{n}(t)\right)$ of real-valued functions, $w^{\prime}=\left(d w_{1} / d t, \cdots, d w_{n} / d t\right), A_{1}$ and $A_{2}$ are real $n \times n$ matrices, $F$ is a continuous function of $3 n$ variables satisfying $F(x)=o(|x|)(|x|=$ $\left.\left(x_{1}^{2}+\cdots+x_{3 n}^{2}\right)^{1 / 2}\right)$ and for any matrix $A$ and vector $v$ by $A v$ we mean the vector $\left(A \cdot v^{t}\right)^{t}$ with $t$ denoting the transpose. We wish to determine when the existence of periodic solutions of the linearized part,

$$
w^{\prime \prime}+A_{1} w^{\prime}+A_{2} w=0,
$$

implies the existence of periodic solutions of (2.1) and the relationships between the periodic solutions of Eq. (2.2) and those of Eq. (2.1).

Using classical results from the theory of ordinary differential equations [3], one can readily verify that Eq. (2.2) has a solution of period $2 \pi \lambda_{0}$ for some $\lambda_{0}>0$ if and only if $\lambda_{0}$ is a characteristic value of the matrix polynomial $A(\lambda)=i \lambda A_{1}+\lambda^{2} A_{2}$ [13]. From the results of $\S 1$ and [13], [1], one might expect Eq. (2.1) to have periodic solutions when $A(\lambda)$ has real characteristic values of odd algebraic multiplicity. However it will be shown in $\S 3$ that unless we make further restrictions on $A_{1}, A_{2}$ and $F,(2.1)$ may have no periodic solutions even though $A(\lambda)$ has only simple characteristic values.

Let us therefore assume $A_{1}$ and $A_{2}$ are of the special form 


$$
\begin{aligned}
A_{1} & =\left[\begin{array}{cc}
0 & \hat{A}_{1} \\
\bar{A}_{1} & 0
\end{array}\right] \\
A_{2} & =\left[\begin{array}{cc}
\hat{A}_{2} & 0 \\
0 & \bar{A}_{2}
\end{array}\right]
\end{aligned}
$$

where $\hat{A_{1}}$ is an $r \times q$ matrix, $\bar{A}_{1}$ a $q \times r$ matrix, $\hat{A}_{2}$ a nonsingular $r \times r$ matrix, and $\bar{A}_{2}$ a $q \times q$ matrix with $0 \leqq q<n$ and $0<r \leqq n$. Thus if

$$
w=(u, v)=\left(u_{1}, \cdots, u_{r}, v_{1}, \cdots, v_{q}\right) .
$$

system (2.1), can be expressed

$$
\begin{gathered}
u^{\prime \prime}+\hat{A}_{1} v^{\prime}+\hat{A}_{2} u+F_{1}\left(u, v, u^{\prime}, v^{\prime}, u^{\prime \prime}, v^{\prime \prime}\right)=0 \\
v^{\prime \prime}+\bar{A}_{1} u^{\prime}+\bar{A}_{2} v+F_{2}\left(u, v, u^{\prime}, v^{\prime}, u^{\prime \prime}, v^{\prime \prime}\right)=0 .
\end{gathered}
$$

We further assume

$$
\begin{aligned}
& F_{1}\left(u,-v,-u^{\prime}, v^{\prime}, u^{\prime \prime},-v^{\prime \prime}\right)=F_{1}\left(u, v, u^{\prime}, v^{\prime}, u^{\prime \prime}, v^{\prime \prime}\right) \\
& F_{2}\left(u,-v,-u^{\prime}, v^{\prime}, u^{\prime \prime},-v^{\prime \prime}\right)=-F_{2}\left(u, v, u^{\prime}, v^{\prime}, u^{\prime \prime}, v^{\prime \prime}\right) .
\end{aligned}
$$

If $\lambda_{1}$ is a positive characteristic value of $A(\lambda)$ let $S\left(\lambda_{1}\right)$ be the set of all different positive characteristic values, $\lambda_{1}, \cdots \lambda_{N}$ of $A(\lambda)$ satisfying $\lambda_{1} / \lambda_{j}=$ integer $(j=1, \cdots, N)$. We define $\mathscr{C}\left(\lambda_{1}\right)=\sum_{\lambda_{j} \in S\left(\lambda_{1}\right)} M\left(\lambda_{j}, A\right)$.

Theorem 2.1. Suppose $S\left(\lambda_{1}\right)$ has $N$ elements, $\mathscr{A}\left(\lambda_{1}\right)$ is odd, and that $|F(x)-F(y)| \leqq h(x, y)|x-y|$ for $x, y \in \boldsymbol{R}^{3 n}$ near zero, where $h(x, y) \rightarrow 0$ as $(x, y) \rightarrow 0$. Then for each sufficiently small $\delta$ there exists a nontrivial periodic solution $w_{\delta}(t)=\left(u_{\hat{\delta}}(t), v_{\delta}(t)\right)$ of Eqs. (2.5), (2.6) with period $2 \pi \lambda_{\delta}$ such that $w_{\delta}(t) \rightarrow 0$ and $\lambda_{\bar{o}} \rightarrow \lambda_{1}$, as $\delta \rightarrow 0$.

Proof. We first introduce a change of variables and set $t=\lambda s$, where $\lambda$ is a real constant to be determined. The resulting system is then

$$
\omega^{\prime \prime}+\lambda A_{1} w^{\prime}+\lambda^{2} A_{2} \omega+\lambda^{2} \hat{F}(\lambda, \omega)=0
$$

where

$$
\hat{F}(\lambda, \omega)=\left(\hat{F}_{1}(\lambda, \omega), \hat{F}_{2}(\lambda, \omega)\right)=\left(F_{1}\left(\omega, \lambda^{-1} \omega^{\prime}, \lambda^{-2} \omega^{\prime \prime}\right), F_{2}\left(\omega, \lambda^{-1} \omega^{\prime}, \lambda^{-2} \omega^{\prime \prime}\right)\right)
$$

and $\omega=(\mu, \nu)=\left(\mu_{1}, \cdots, \mu_{r}, \nu_{1}, \cdots, \nu_{q}\right)$. It suffices to determine the $2 \pi$ periodic solutions of (2.7) as they correspond to $2 \pi \lambda$ periodic solution of (2.1).

Even $2 \pi$ periodic functions are of the form $\mu=\mu_{0}+\mu^{*}$, where $\mu^{*}=1 / 2 \pi \int_{0}^{2 \pi} \mu(s) d s$, the mean value of $\mu$, and $\mu_{0}$ is of mean value 
zero. Thus Eq. (2.7) can be expresed equivalently as

$$
\begin{gathered}
\hat{A}_{2} \mu^{*}+\frac{1}{2 \pi} \int_{0}^{2 \pi} \hat{F}_{1}\left(\lambda, \mu_{0}(s)+\mu^{*}, \nu(s)\right) d s=0 \\
\mu_{0}^{\prime \prime}+\lambda \hat{A}_{1} \nu^{\prime}+\lambda^{2}\left[\hat{A}_{2} \mu_{0}+\hat{F}_{1}\left(\lambda, \mu_{0}+\mu^{*}, \nu\right)\right. \\
\left.-\frac{1}{2 \pi} \int_{0}^{2 \pi} \hat{F}_{1}\left(\lambda, \mu_{0}(s)+\mu^{*}, \nu(s)\right) d s\right]=0 \\
\nu^{\prime \prime}+\lambda \bar{A}_{1} \mu_{0}^{\prime}+\lambda^{2}\left[\bar{A}_{2} \nu+\hat{F}_{2}\left(\lambda, \mu_{0}+\mu^{*}, \nu\right)\right]=0 .
\end{gathered}
$$

To apply Theorem 1.8 we introduce the appropriate Banach spaces. Let $\mathscr{C}_{1}$ be the space of twice continuously differentiable, $2 \pi$ periodic, even $r$-vector functions of mean value zero and $\mathscr{C}_{2}$ the space of twice continuously differentiable, $2 \pi$ periodic, odd $q$-vector functions.

Then let $\mathscr{C}$ be the Banach space defined by $\mathscr{C}=\mathscr{C}_{1} \times \mathscr{C}_{2}$ with the norm

$$
\||\omega|\|=\left\|\left|\left(\omega_{1}, \cdots, \omega_{n}\right) \|\right|=\max _{\substack{j=1, \ldots, n \\ k=0,1,2}}\left\{\left\|\omega_{j}^{(k)}\right\|\right\}\right.
$$

where $\left\|\omega_{j}^{(k)}\right\|=\sup _{0 \leqq t \leqq 2 \pi}\left|\omega_{j}^{(k)}(t)\right|$. Note that there is a $c \geqq 0$ such that for all $\left.\omega \in \mathscr{C},\|\omega \mid\| \leqq c \max _{j=1, \cdots, n}\left\|\omega_{j}^{\prime \prime}\right\|\right\}$. Indeed if $f(t)$ is a twice continuously differentiable $2 \pi$ periodic function of mean value zero then there exist $t_{1}$ and $t_{2}$ such that $f\left(t_{1}\right)=f^{\prime}\left(t_{2}\right)=0$. Therefore for each $t$

$$
|f(t)|=\left|f(t)-f\left(t_{1}\right)\right| \leqq\left|t-t_{1}\right|\left|f^{\prime}(\bar{t})\right| \leqq\left|t-t_{1}\right|\left|\bar{t}-t_{2}\right|\left|f^{\prime \prime}\left(t_{0}\right)\right|,
$$

where $\bar{t}$ lies between $t_{1}$ and $t$, and $t_{0}$ lies between $\bar{t}$ and $t_{2}$. Hence $\|f(t)\| \leqq 2 \pi\left\|f^{\prime}(t)\right\| \leqq 4 \pi^{2}\left\|f^{\prime \prime}(t)\right\|$. Thus

$$
\|\omega\| \| \leqq c \max _{j=1, \cdots, n}\left\{\left\|\omega_{j}^{\prime \prime}\right\|\right\} .
$$

Let $H_{1}$ be the Hilbert space of absolutely continuous, $2 \pi$ periodic, even $r$-vector functions of mean value zero such that $\int_{0}^{2 \pi}\left(\mu_{0}^{\prime}(s)^{2}\right) d s<\infty$ and $H_{2}$ the Hilbert space of absolutely continuous, $2 \pi$ periodic, odd $q$-vector functions such that $\int_{0}^{2 \pi}\left(\nu^{\prime}(s)^{2}\right) d s<\infty$. Let $H=H_{1} \times H_{2}$ with inner product defined by $\langle x, y\rangle=\int_{0}^{2 \pi} x^{\prime}(s) \cdot y^{\prime}(s) d s, x=\left(\mu_{0}, \nu\right)$. And lastly let $X^{*}$ be the space of the $\mu^{*}$ with Euclidean norm. For convenience we express $\left(\mu_{0}+\mu^{*}, \nu\right)$ as $x+\mu^{*}$.

$\hat{A}_{2}$ is nonsingular and we consider now finding solutions of the operator equations

$$
\mu^{*}+\hat{A}_{2}^{-1}\left[\frac{1}{2 \pi} \int_{0}^{2 \pi} \hat{F}_{1}\left(\lambda, \mu_{0}(s)+\mu^{*}, \nu(s)\right) d s\right]=0
$$


(2.12)

$$
x-\mathscr{A}(\lambda) x-\lambda^{2} \mathscr{F}\left(\lambda, x+\mu^{*}\right)=0
$$

in $\boldsymbol{R} \times X^{*} \times \mathscr{C}$ where $\mathscr{A}(\lambda)=\lambda \mathscr{A}_{1}+\lambda^{2} \mathscr{A}_{2}$ and for $\lambda \in \boldsymbol{R}$ and $x \in$ $\mathscr{C}, \mathscr{A}_{1} x, \mathscr{A}_{2} x$ and $\mathscr{F}(\lambda, x)$ are defined to be the unique element in $\mathscr{C}$ such that

$$
\left\langle\mathscr{A}_{1} x, y\right\rangle=\int_{0}^{2 \pi} A_{1} x^{\prime}(s) \cdot y(s) d s,\left\langle\mathscr{A}_{2} x, y\right\rangle=\int_{0}^{2 \pi} A_{2} x(s) \cdot y(s) d s
$$

and

$$
\left\langle\mathscr{F}\left(\lambda, x+\mu^{*}\right), y\right\rangle=\int_{0}^{2 \pi} F\left(\lambda, x(s)+\mu^{*}\right) \cdot y(s) d s,
$$

for all $y \in H$.

It is clear that if $\left(\lambda, x+\mu^{*}\right)$ is a solution of Eqs. (2.11) and (2.12) then it is a solution of Eq. (2.7) as we have

$$
\int_{0}^{2 \pi}\left(x^{\prime \prime}+\lambda A_{1} x^{\prime}+\lambda^{2} A_{2} x+\lambda^{2} \hat{F}\left(\lambda, x+\mu^{*}\right)\right), \quad y=0
$$

for all $y \in H$.

Now $\mathscr{A}_{1}, \mathscr{A}_{2}$ and $\mathscr{F}$ satisfy the equations

$$
d \cdot \mathscr{A}_{1} x / d s=-A_{1} x, d^{2} \mathscr{A _ { 2 }} x / d s^{2}=-A_{2} x
$$

and

$$
\partial^{2} \mathscr{F}\left(\lambda, x+\mu^{*}\right) / \partial s^{2}=-\hat{F}\left(\lambda, x+\nu^{*}\right)+\frac{1}{2 \pi} \int_{0}^{2 \pi} \hat{F}_{1} .
$$

Thus an application of the Ascoli-Arzela theorem will verify that $\mathscr{A}_{1}$ and $\mathscr{A}_{2}$ are compact maps of $\mathscr{C}$ into $\mathscr{C}$. By Eq. (2.10a) it follows $\left(\hat{A}_{2}^{-1}\left[1 / 2 \pi \int_{0}^{2 \pi} \hat{F}_{1}\right], \mathscr{F}\right)$ is a continuous nonlinear map of $\boldsymbol{R} \times X^{*} \times \mathscr{C}$ into $X^{*} \times \mathscr{C}$ satisfying Eq. (1.11).

In the course of proving theorem 1.5 it was shown that $I-\lambda_{0}(W+V)$ is a closed Fredholm operator of index zero whenever $A_{1}, \cdots, A_{n}$ are compact. Thus by Theorem 1.8 the proof of Theorem Theorem 2.1 will be complete once we have shown that $\lambda_{1}$ is a characteristic value of odd algebraic multiplicity of the linearized part of (2.11), (2.12),

$$
\left[\begin{array}{cc}
0 & 0 \\
0 & \mathscr{A}(\lambda)
\end{array}\right]
$$

(which is a mapping of $X^{*} \times \mathscr{C}$ into $X^{*} \times \mathscr{C}$ ). It suffices to show $M\left(\lambda_{1}, \mathscr{C}\right)$ is odd. To this end we prove the lemma 
LEMMA 2.2. Under the hypotheses of Theorem 2.1, $M\left(\lambda_{1}, \mathscr{A}\right)$ is odd.

Proof. If $\lambda_{k} \in S\left(\lambda_{1}\right)$ and $(a+i b, c+i d)$ with $\dot{\alpha}, b \in \boldsymbol{R}^{r}$ and $c, d \epsilon$ $\boldsymbol{R}^{q}$ is a characteristic vector corresponding to $\lambda_{k}$, then by equating components we have the equalities

$$
\begin{aligned}
& \lambda_{k}^{2} \hat{A}_{2} a-\lambda_{k} \hat{A}_{1} d=a \\
& \lambda_{k}^{2} \bar{A}_{2} d+\lambda_{k} \bar{A}_{1} a=d \\
& \lambda_{k}^{2} \hat{A}_{2} b+\lambda_{k} \hat{A}_{1} c=b \\
& \lambda_{k}^{2} \bar{A}_{2} c-\lambda_{k} \bar{A}_{1} b=c .
\end{aligned}
$$

On the other hand it is clear that for all integers $p>0$

$$
\begin{aligned}
& \mathscr{A}_{1}(\alpha \cos p s, \beta \sin p s)=1 / p\left(\hat{A}_{1} \beta \cos p s,-\bar{A}_{1} \alpha \sin p s\right) \\
& \mathscr{A}_{2}(\alpha \cos p s, \beta \sin p s)=1 / p^{2}\left(\hat{A}_{2} \alpha \cos p s, \bar{A}_{2} \beta \sin p s\right) .
\end{aligned}
$$

Thus if $p_{k}=\lambda_{1} / \lambda_{k},\left(b \cos p_{k} s, c \sin p_{k} s\right)$ is a characteristic vector corresponding to $\lambda_{1}$ if and only if $(b, c)$ is a characteristic vector corresponding to the characteristic value $\lambda_{k}$ of $\lambda \widetilde{A}_{1}+\lambda^{2} A_{2}$ where

$$
\widetilde{A}_{1}=\left[\begin{array}{cc}
0 & \hat{A}_{1} \\
-\bar{A}_{1} & 0
\end{array}\right] \text {. }
$$

Moreover from Eqs. (2.15) and (2.16) it follows that for

$$
L=\left[\begin{array}{ll}
0 & \mathscr{A}_{2} \\
I & \mathscr{A}_{1}
\end{array}\right]
$$

if $(y, x) \in \mathscr{C} \times \mathscr{C}$ is an element of $N_{\infty}\left(I-\lambda_{1} L\right)$ then $y$ and $x$ must be of the form

$$
y=\sum_{j=1}^{N}\left(\alpha_{j} \cos p_{j} s, \beta_{j} \sin p_{j} s\right) \quad p_{j}=\lambda_{1} / \lambda_{j}
$$

and

$$
x=\sum_{j=1}^{N}\left(\gamma_{j} \cos p_{j} s, \delta_{j} \sin p_{j} s\right) \quad p_{j}=\lambda_{1} / \lambda_{j}
$$

and the coefficient vector $\left(\alpha_{j}, \beta_{j}, \gamma_{j}, \delta_{j}\right)$ for $j=1, \cdots, N$ is an element of $N_{\infty}\left(I-\lambda_{j} Q\right)$, where

$$
Q=\left[\begin{array}{cc}
0 & A_{2} \\
I & \widetilde{A}_{1}
\end{array}\right]
$$

Conversely, if $(\alpha, \beta, \gamma, \delta) \in N_{\infty}\left(I-\lambda_{j} Q\right)$ then $\left(\alpha \cos p_{j} s, \beta \sin p_{j} s\right.$, $\left.\gamma \cos p_{j} s, \delta \sin p_{j} s\right) \in N_{\infty}\left(I-\lambda_{1} L\right)$. Hence 


$$
\operatorname{dim} N_{\infty}\left(I-\lambda_{1} L\right)=\sum_{j=1}^{N} \operatorname{dim} N_{\infty}\left(I-\lambda_{j} Q\right) .
$$

For $j=1, \cdots, N, \operatorname{dim} N_{\infty}\left(I-\lambda_{j} Q\right)$ is equal to the multiplicity of $\lambda_{j}$ as a root of $\operatorname{det}|I-A(\lambda)|=0$. Indeed by the remark preceding Theorem 1.5 it follows that $\operatorname{dim} N_{\infty}\left(I-\lambda_{j} Q\right)$ is equal to the multiplicity of $\lambda_{j}$ as a root of $\operatorname{det}\left|I-\lambda^{2} A_{2}-\lambda \widetilde{A}_{1}\right|=0$. It is easily verified that if $P$ is a nonsingular square matrix we have the formula

$$
\begin{aligned}
\operatorname{det}\left|\begin{array}{ll}
P & Q \\
R & S
\end{array}\right| & =\operatorname{det}\left|\left[\begin{array}{cc}
I & 0 \\
-R P^{-1} & I
\end{array}\right] \cdot\left[\begin{array}{ll}
P & Q \\
R & S
\end{array}\right]\right| \\
& =\operatorname{det}|P| \cdot \operatorname{det}\left|S-R P^{-1} Q\right| .
\end{aligned}
$$

Thus for all but a finite number of $\lambda$ we have

$$
\begin{aligned}
\operatorname{det} & \left|I-\lambda^{2} A_{2}-\lambda \widetilde{A}_{1}\right| \\
& =\operatorname{det}\left|I-\lambda^{2} \hat{A}_{2}\right| \cdot \operatorname{det}\left|\left(I-\lambda^{2} \bar{A}_{2}\right)-\lambda \bar{A}_{1}\left(I-\lambda^{2} \hat{A}_{2}\right)^{-1} \lambda \hat{A}_{1}\right| \\
& =\operatorname{det}|I-A(\lambda)| .
\end{aligned}
$$

However they are polynomials and so

$$
\operatorname{det}\left|I-\lambda^{2} A_{2}-\lambda \widetilde{A}_{1}\right| \equiv \operatorname{det}|I-A(\lambda)| \text {. }
$$

Hence $\operatorname{dim} N_{\infty}\left(I-\lambda_{j} Q\right)=M\left(\lambda_{j}, A\right)$. Therefore by (2.17) $\operatorname{dim} N_{\infty}\left(I-\lambda_{1} L\right)$ is odd.

With Lemma 2.2 the proof of Theorem 2.1 is complete.

If $F$ depends on $w^{\prime \prime}$ then $\mathscr{F}$ need not be completely continuous and we cannot establish the existence of large periodic solutions of (2.1). Therefore we now consider the system of ordinary differential equations.

$$
w^{\prime \prime}+A_{1} w^{\prime}+A_{2} w+F\left(w, w^{\prime}\right)=0
$$

where $A_{1}$ and $A_{2}$ are as described by (2.3) and (2.4), and $F$ is a continuous function of $2 n$ variables satisfying

$$
\begin{aligned}
F(x) & =o(|x|), F=\left(F_{1}, F_{2}\right), F_{1}\left(u,-v,-u^{\prime}, v^{\prime}\right) \\
& =F_{1}\left(u, v, u^{\prime}, v_{\triangleleft}\right), F_{2}\left(u,-v,-u^{\prime}, v^{\prime}\right)=-F_{2}\left(u, v, u^{\prime}, v^{\prime}\right) .
\end{aligned}
$$

To characterize sets of nontrivial periodic solutions of (2.19) let $E$ be the set of nontrivial $2 \pi$ periodic solutions with $\lambda>0$, of

$$
\omega^{\prime \prime}+\lambda A_{1} \omega^{\prime}+\lambda^{2} A_{2} \omega+\lambda^{2} \hat{F}(\lambda, \omega)=0
$$

in $\boldsymbol{R} \times X^{*} \times \mathscr{C}$, where $\hat{F}(\lambda, \omega)=F\left(\omega, \lambda^{-1} \omega^{\prime}\right)$. Clearly if $\left(\lambda, \mu^{*}, x\right) \in E$ then $x(t / \lambda)+\mu^{*}$ is a $2 \pi \lambda$ periodic solution of $(2.20)$. The closure 
of $E$ in $R \times X^{*} \times \mathscr{C}$ will be denoted by $\bar{E}$.

We note that $\hat{F}(\lambda, \omega)$ is undefined for $\lambda=0$ so that care must be taken when dealing with solutions with $\lambda$ near zero.

THEOREM 2.3. Let $\lambda_{1}$ be a characteristic value of $A(\lambda)$ and suppose $S\left(\lambda_{1}\right)$ has $N$ elements and $\mathscr{M}\left(\lambda_{1}\right)$ is odd. Then $\bar{E}$ contains a maximal set of solutions, $C_{\lambda_{1}}$, such that $C_{\lambda_{1}}$ meets $\left(\lambda_{1}, 0,0\right), C_{\lambda_{1}}$ is closed and connected in $R \times X^{*} \times \mathscr{C}$ and either

(i) $C_{\lambda_{1}}$ meets a point $\left(\lambda_{0}, 0,0\right)$ such that for some positive integer $m, \lambda_{0} / m$ is a positive characteristic value of $A(\lambda), \lambda_{0} \notin S\left(\lambda_{1}\right)$, and $\mathscr{L}\left(\lambda_{0} / m\right)$ is odd or

(ii) $C_{\lambda_{1}}$ is unbounded in $\boldsymbol{R} \times X^{*} \times \mathscr{C}$ or

(iii) for each $\lambda_{1}>\varepsilon>0, C_{\lambda_{1}}$ meets a point of the form $\left(\varepsilon, \mu_{\varepsilon}^{*}, x_{\varepsilon}\right)$.

Proof. Let $\mathscr{A}_{1}, \mathscr{A}_{2}$ and $\mathscr{F}\left(\lambda, x+\mu^{*}\right)$ be defined by (2.13) and (2.14). As $\partial^{2} \mathscr{F}\left(\lambda, x+\mu^{*}\right) / \partial s^{2}=-\hat{F}\left(\lambda, x+\mu^{*}\right)+1 / 2 \pi \int_{0}^{2 \pi} \hat{F}_{1}$, an application of the Ascoli-Arzela theorem will show that

$$
\left(A_{2}^{-1}\left[\frac{1}{2 \pi} \int_{0}^{2 \pi} \hat{F}_{1}\right], \mathscr{F}\right)
$$

is completely continuous. The positive characteristic values of $\mathscr{A}(\lambda)$ are all of the form $\beta m$ where $m$ is a positive integer and $\beta$ is a positive characteristic value of $A(\lambda)$. Thus by repeating the proof of Theorem 2.1 and applying Theorems 1.6 and 1.7 to Eqs. (2.11) and (2.12) on $\Omega_{j, \varepsilon}=I_{j, \varepsilon} \times S_{j}$ for $j=1, \cdots, 0<\varepsilon<\lambda_{1}$

$$
I_{j, \varepsilon}=\{\lambda \mid \varepsilon<\lambda<j\}, S_{j}=\left\{\left(\mu^{*}, x\right) \mid\left\|\left(\mu^{*}, x\right)\right\|<j\right\},
$$

there exists a unique maximal closed connected set $C_{j, \varepsilon} \subset \bar{E}$ meeting $\left(\lambda_{1}, 0\right)$ such that $C_{j, \varepsilon} \subset \bar{\Omega}_{j, \varepsilon}, C_{j, \varepsilon}$ either meets $\partial \Omega_{j, \varepsilon}$ or meets $\left(\lambda_{0}, 0,0\right)$, where $\lambda_{0}$ is a positive characteristic value of $\mathscr{A}(\lambda)$ and $M\left(\lambda_{0}, \mathscr{A}\right)$ is odd. As the union of connected sets containing a common point is connected, there is a unique maximal closed connected subset $C_{\lambda_{1}}$ of $\bar{E}$ meeting $\left(\lambda_{1}, 0\right)$. Hence $\cup C_{j, \varepsilon} \subset C_{\lambda_{1}}$ and the theorem follows.

If we make the further assumption that $F$ does not depend on $w^{\prime}$ then $\hat{F}$ will be independent of $\lambda$ and we no longer have to be concerned with the existence of bounded solutions of (2.1) with periods tending to zero.

THEOREM 2.4. Let $\lambda_{1}$ be as in Theorem 2.2 and suppose $F=$ $F(w)$. Then there exists a maximal subset $C_{\lambda_{1}}$ of $\bar{E}$ such that $C_{\lambda_{1}}$ is closed and connected in both $\boldsymbol{R} \times X^{*} \times \mathscr{C}$ and $\boldsymbol{R} \times X^{*} \times H$ and either 
(i) $C_{\lambda_{1}}$ is unbounded in both $\boldsymbol{R} \times X^{*} \times \mathscr{C}$ and $\boldsymbol{R} \times X^{*} \times H$ or

(ii) $C_{\lambda_{1}}$ meets a point $\left(\lambda_{0}, 0,0\right)$ such that for some positive integer $m, \lambda_{0} / m$ is a positive characteristic value of $A(\lambda), \lambda_{0} \notin S\left(\lambda_{1}\right)$ and $\mathscr{L C}\left(\lambda_{0} / m\right)$ is odd.

Proof. It it is readily verified that $\mathscr{A}_{1}, \mathscr{A}_{2}$ and $\mathscr{F}$ are completely continuous maps of $H \rightarrow H$ and $R \times X^{*} \times H \rightarrow H$ (cf. [1], [2, pp. 155156]). Moreover the algebraic multiplicity of $\lambda_{1}$ of $\mathscr{A}(\lambda)$ is same for $\mathscr{A}(\lambda)$ as map of $\mathscr{C} \rightarrow \mathscr{C}$ and of $H \rightarrow H$. Thus arguing as in the proof of Theorems 2.1 and 2.3 and applying Theorems 1.5 and 1.7 to (2.11), (2.12) on $R \times X^{*} \times \mathscr{C}$ or $R \times X^{*} \times H$ there exists a maximal connected set of nontrivial solutions of (2.20) whose closure, $S$, meets $\left(\lambda_{1}, 0,0\right)$ and is either unbounded in both $R \times X^{*} \times \mathscr{C}$ and $\boldsymbol{R} \times X^{*} \times H$ or meets a $\left(\lambda_{0}, 0,0\right)$ where $\lambda_{0} \in r(\mathscr{A})$ and $M\left(\lambda_{0}, \mathscr{A}\right)$ is odd. Let $C_{\lambda_{1}}$ be the maximal closed connected subset of $S$ meeting $\left(\lambda_{1}, 0,0\right)$ and containing those solutions $\left(\lambda, \mu^{*}, x\right)$ such that $\lambda \geqq 0$. If $C_{\lambda_{1}}$ meets a point of the form $\left(\hat{\lambda}, \mu_{1}^{*}, 0\right), u_{1}^{*} \neq 0$, then $C_{\lambda_{1}}$ must contain all points of the form $\left(\lambda, \mu_{1}^{*}, 0\right), 0 \leqq \lambda$, and so $C_{\lambda_{1}}$ satisfies (i). So suppose $C_{\lambda_{1}}$ does not meet any points of the form $\left(\hat{\lambda}, \mu_{1}^{*}, 0\right)$. Then $C_{\lambda_{1}}$ cannot meet any points of the form $\left(0, \mu^{*}, x\right)$. Indeed, suppose $C_{\lambda_{1}}$ met a point $\left(0, \mu^{*}, x\right)$. If $x \neq 0$, then as $\left(0, \mu^{*}, x\right)$ is a solution of Eq. (2.20) we have $\left(x+\mu^{*}\right)^{\prime \prime} \equiv 0$, which is impossible if $x \neq 0$. On the other hand $x=0$ implies $\mu^{*}=0$ and so $\lambda=0$ is a bifurcation point of (2.11), (2.12). However as $\lambda=0$ is not a characteristic value of the linearized part of (2.11), (2.12) it cannot be a bifurcation point. Therefore $C_{\lambda_{1}}$ does not meet any points of the form $\left(0, \mu^{*}, x\right)$. By the complete continuity of the operators $S$ is locally compact. Thus unless $C_{\lambda_{1}}$ is unbounded there exists a $\delta>0$ such that if $\left(\lambda, \mu^{*}, x\right) \in C_{\lambda_{1}}, \lambda>\delta$. Hence either $C_{\lambda_{1}}$ is unbounded or $C_{\lambda_{1}}=S$ and $C_{\lambda_{1}}$ satisfies (ii).

With a rather strong condition on the derivative of the nonlinear operators we can show the existence of an unbounded set of solutions $C_{\lambda}$. To this end we first show

Lemma 2.5. Let $T(\lambda, z) \in C^{1}$ be a completely continuous map of $\boldsymbol{R} \times X^{*} \times \mathscr{C} \rightarrow X^{*} \times \mathscr{C}$ such that $T(\lambda, z)$ has the same period as $(\lambda, z)$. Suppose $T\left(\lambda_{0}, z_{0}\right)=z_{0}$ and every $z \in N_{\infty}\left(I-T_{z}\left(\lambda_{0}, z_{0}\right)\right)$ is $2 \pi / m$ periodic where $m$ is a positive integer. Then all solutions of

$$
T(\lambda, z)=z
$$

in a neighborhood of $\left(\lambda_{0}, z_{0}\right)$ are $2 \pi / m$ periodic.

Proof. As $T(\lambda, z)$ is completely continuous so is the derivative 
$T_{z}\left(\lambda_{0}, z_{0}\right)=T^{\prime}[12$, p. 51]. Thus by the Fredholm theory [11, pp. 179-190, 219], $\alpha\left(I-T^{\prime}\right)=p<\infty, \operatorname{dim} N_{p}\left(I-T^{\prime}\right)<\infty$ and $X^{*} \times \mathscr{C}=$ $N_{p}\left(I-T^{\prime}\right) \oplus R_{p}\left(I-T^{\prime}\right)$. Let $P$ be the canonical projection of $X^{*} \times$ $\mathscr{C}$ onto $R_{p}\left(I-T^{\prime}\right)$. Then finding solutions of (2.21) is equivalent to solving

$$
\begin{aligned}
& u-P T(\lambda, u+v)=0 \\
& v-(I-P) T(\lambda, u+v)=0
\end{aligned}
$$

where $u \in R_{p}\left(I-T^{\prime}\right)$ and $v \in N_{p}\left(I-T^{\prime}\right)$. By the implicit function theorem [5, pp. 265] near $\left(\lambda_{0}, v_{0}\right)$ there exists a unique function $u=$ $u(\lambda, v)$ such that $u(\lambda, v) \equiv P T(\lambda, u(\lambda, v)+v)$. Moreover $u(\lambda, v)$ is found by an iteration of $2 \pi / m$ functions and so is itself $2 \pi / m$ periodic. Thus all solutions of (2.21) near $\left(\lambda_{0}, z_{0}\right)$ must be of the form $v+u(\lambda, v)$ and the result follows.

THEOREM 2.6. Let $\lambda_{1}<\cdots<\lambda_{K}$ be the real characteristic values of $A(\lambda)$. Suppose there exist $H$ consecutive positive characteristic values $\lambda_{k+1}<\cdots \lambda_{k+H}, 1 \leqq k \leqq K$ such that $\sum_{j=1}^{H} M\left(\lambda_{k+j}, A\right)$ is odd, $p \lambda_{j} \notin\left[\lambda_{k+1}, \lambda_{k+H}\right](p=1, \cdots ; j \leqq k), \lambda_{k+H}<2 \lambda_{k+1} S\left(\lambda_{k+j}\right)=\left\{\lambda_{k+j}\right\}(j=1, \cdots, H)$ and for $0<\lambda_{j} \notin\left[\lambda_{k+1}, \lambda_{k+H}\right], \mathscr{C l}\left(\lambda_{j}\right)$ is even.

(i) If $F=F(w), \bar{E}$ contains a maximal set of solutions $C_{\lambda_{k+i}}$ such that, $1 \leqq i \leqq H, C_{\lambda_{k+i}}$ meets $\left(\lambda_{k+i}, 0,0\right), C_{\lambda_{k+i}}$ is closed and connected in both $\boldsymbol{R} \times X^{*} \times \mathscr{C}$ and $\boldsymbol{R} \times X \times H$ and either $C_{k_{k+i}}$ is unbounded in both $\boldsymbol{R} \times X^{*} \times \mathscr{C}$ and $\boldsymbol{R} \times X^{*} \times H$ or meets a point $\left(\lambda_{j}, 0,0\right)$ such that $\lambda_{j} / m$ is a positive characteristic value of $A(\lambda)$ for some integer $m>1$ and $\mathscr{C}\left(\lambda_{j} / m\right)$ is odd.

(ii) If in addition $F \in C^{1}$ and at any nontrivial solution $\left(\lambda_{0}, \mu_{0}^{*}, x_{0}\right)$ of $(2.11),(2.12)$, the derivitive with respects to $\left(\mu_{0}^{*}, x_{0}\right)$

$$
\psi=\left[\begin{array}{cc}
I+A_{2 m}^{-1}\left[\frac{1}{2 \pi} \int_{0}^{2 \pi} F_{1}^{\prime}\left(x_{0}(s)+\mu_{0}^{*}\right) d s\right] & 0 \\
0 & I-\mathscr{A}\left(\lambda_{0}\right)-\lambda_{0} \mathscr{F}^{\prime}\left(x_{0}+\mu_{0}^{*}\right)
\end{array}\right]
$$

is such that $\left(\mu^{*}, x(s)\right) \in N_{\infty}(\Psi)$ implies $x(s)$ has the same period as $x_{0}(s)$, then $C_{\lambda_{k+j}}$ is unbounded.

Proof. The characteristic values $\lambda_{k+1}, \cdots, \lambda_{k+I I}$ are the only characteristic values of $\mathscr{A}(\lambda)$ in $\left[\lambda_{k+1}, \lambda_{k+I I}\right]$ and by Lemma 2.2 $\sum_{j=1}^{I I} M\left(\lambda_{k+j}, \mathscr{A}\right)$ is odd. Thus arguing as in the proof of Theorem 2.4 there exists a maximal subset $C_{\lambda_{k+i}}$ of $\bar{E}$ with the properties of (i). To complete the proof we suppose that $F$ is as described in (ii) and $C_{\lambda_{k+2}}$ is bounded and meets a points of the form $\left(\lambda_{1}, 0,0\right)$ where $\lambda_{1} / m$ is a characteristic value of $A(\lambda)$ and $m$ is an integer $>1$. As $\mathscr{A}(\lambda)$ and $\mathscr{F} \operatorname{map} 2 \pi / h$ periodic functions into $2 \pi / h$ periodic functions 
it follows from Lemma 2.5 that $C_{\lambda_{k+i}}$ contains both $2 \pi$ and $2 \pi / m$ periodic solutions. By the connectedness of $C_{\lambda_{k+i}}$ there must exist a $2 \pi / m$ periodic solution $\left(\lambda_{0}, \mu_{0}^{*}, x_{0}\right) \in C_{\lambda_{k+i}}$ such that every neighborhood of $\left(\lambda_{0}, \mu_{0}^{*}, x_{0}\right)$ contains $2 \pi$ periodic solutions of (2.11), (2.12). But by our hypotheses and Lemma 3.5 this is impossible hence the result.

REMARK. Suppose (2.1) has the form

$$
w^{\prime \prime}+A_{2} w+F\left(w, w^{\prime}, w^{\prime \prime}\right)=0
$$

where $A_{2}$ is an $n \times n$ matrix and $F\left(-w, w^{\prime},-w^{\prime \prime}\right)=-F\left(w, w^{\prime}, w^{\prime \prime}\right)$ then if $\mathscr{C}$ and $H$ are replaced by $\mathscr{C}_{2}$ and $H_{2}$ proofs similar to those of Theorems 2.1, 2.3, and 2.4 will show that these theorems are also true for (2.22)

3. A counterexample. We give an example to show that unless some additional restrictions are imposed the system $x^{\prime \prime}+A_{1} x^{\prime}+A_{2} x+$ $F(x)=0$ may fail to have any periodic solutions even though $\lambda^{2} A_{2}+$ $i \lambda A_{1}$ has only simple eigenvalues.

Consider the system

$$
\begin{aligned}
& x^{\prime \prime}+3 y^{\prime}+4 x+y^{3}=0 \\
& y^{\prime \prime}-3 x^{\prime}+4 y-x^{3}=0 .
\end{aligned}
$$

The characteristic equation

$$
\operatorname{det}\left|\left[\begin{array}{ll}
4 & 0 \\
0 & 4
\end{array}\right]+i \beta\left[\begin{array}{rr}
0 & 3 \\
-3 & 0
\end{array}\right]-\beta^{2}\left[\begin{array}{ll}
1 & 0 \\
0 & 1
\end{array}\right]\right|=0
$$

has four distinct roots $\pm 1, \pm 4$. Hence $\lambda=1,1 / 4$, is a simple characteristic value of $\lambda^{2} A_{2}+i \lambda A_{1}$. On the other hand the system (3.1), (3.2), has no nontrivial periodic solutions. Suppose $(x(t), y(t))$ is a periodic solution of (3.1), (3.2) of period $a$. Multiply (3.1) by $y$ and (3.2) by $x$. Then integrate the resulting equations by parts over $[0, a]$, noting $\int_{0}^{a} x^{\prime} x=\int_{0}^{a} y^{\prime} y=y^{2} /\left.2\right|_{0} ^{a}=0$. If we now subtract we obtain

$$
\int_{0}^{a} y^{4}+x^{4}=0 .
$$

This implies $y=x \equiv 0$ and hence there do not exist nontrivial periodic solutions of (3.1), (3.2).

After this work was completed, G. I. Ize's doctoral dissertation [9] was brought to our attention. In it he gives an alternate, analytic, definition of the algebraic multiplicity of a characteristic value and with his definition proves theorems similar to our Theorem 1.5. 


\section{REFERENCES}

1. M. S. Berger, On periodic solutions of second order Hamiltonian systems (I), J. Math. Anal. Appl., 29 (1970), 512-522.

2. M. S. Berger and M. S. Berger Perspectives in Nonlinearity. An Introduction to Nonlinear Analysis, Benjamin, New York, 1968.

3. E. A. Coddington and N. Levinson, The Theory of Ordinary Differential Equations, McGraw-Hill Book Co., New, York, 1955.

4. E. N. Dancer, On the structure of solutions of non-linear eigenvalue problems, Indiana U. Math. J., 23 (1974), 1069-1076.

5. J. Dieudonné, Fundations of Modern Analysis, Academic Press, New York, 1960.

6. R. Edwards, Functional Analysis, Theory and Applications, Holt, Rinehart, and Winston, New York, 1965.

7. P. R. Halmos, Finite-Dimensional Vector Spaces, Second Edition, D. Van Nostrand Company, Inc., Princeton, 1958.

8. — Naive Set Theory, D. Van Nostrand Company, Inc., Princeton, 1966.

9. G. I. Ize, Bifurcation theory for Fredholm operators, Doctoral Dissertation, New York University, April, 1974.

10. P. H. Rabinowitz, Some global results for nonlinear eigenvalue problems, J. Functional Analysis, 7 (1971), 487-513.

11. F. Riesz and B. Sz-Nagy, Functional Analysis, Frederick Ungar Publishing Co., New York, 1955.

12. M. M. Vainberg, Variational Methods for the Study of Nonlinear Operators, GITTL, Moscow, 1956; English trans., Holden-Day, San Francisco, Calif., 1964.

13. D. Westreich, Periodic solutions of second order non-Lagrangian systems, Israel J. Math., 16 (1973), 279-286.

14. - Bifurcation of operator equations with unbounded linerized part, Pacific J. Math., 57 (1975), 611-618.

Received September 2, 1975 and in revised form September 9, 1976.

Ben-Gurion University of THE NegeV

BeEr-Sheva, IsRael 


\section{PACIFIC JOURNAL OF MATHEMATICS}

\section{EDITORS}

RICHARD ARENS (Managing Editor)

University of California

Los Angeles, California 90024

\section{J. DugunduI}

Department of Mathematics University of Southern California Los Angeles, California 90007

D. Gilbarg and J. Milgram

Stanford University

Stanford, California 94305

\author{
R. A. Beaumont \\ University of Washington \\ Seattle, Washington 98105
}

\section{ASSOCIATE EDITORS}
B. H. NeUmanN
F. WOLF
K. YosHIDA

\section{SUPPORTING INSTITUTIONS}

\author{
UNIVERSITY OF BRITISH COLUMBIA \\ CALIFORNIA INSTITUTE OF TECHNOLOGY \\ UNIVERSITY OF CALIFORNIA \\ MONTANA STATE UNIVERSITY \\ UNIVERSITY OF NEVADA \\ NEW MEXICO STATE UNIVERSITY \\ OREGON STATE UNIVERSITY \\ UNIVERSITY OF OREGON \\ OSAKA UNIVERSITY
}

\author{
UNIVERSITY OF SOUTHERN CALIFORNIA \\ STANFORD UNIVERSITY \\ UNIVERSITY OF HAWAII \\ UNIVERSITY OF TOKYO \\ UNIVERSITY OF UTAH \\ WASHINGTON STATE UNIVERSITY \\ UNIVERSITY OF WASHINGTON \\ AMERICAN MATHEMATICAL SOCIETY
}

The Supporting Institutions listed above contribute to the cost of publication of this Journal, but they are not owners or publishers and have no responsibility for its content or policies.

Mathematical papers intended for publication in the Pacific Journal of Mathematics should be in typed form or offset-reproduced, (not dittoed), double spaced with large margins. Please do not use built up fractions in the text of your manuscript. You may however, use them in the displayed equations. Underline Greek letters in red, German in green, and script in blue. The first paragraph or two must be capable of being used separately as a synopsis of the entire paper. Items of the bibliography should not be cited there unless absolutely necessary, in which case they must be identified by author and Journal, rather than by item number. Manuscripts, in triplicate, may be sent to any one of the editors. Please classify according to the scheme of Math. Reviews, Index to Vol. 39. All other communications should be addressed to the managing editor, or Elaine Barth, University of California, Los Angeles, California, 90024.

The Pacific Journal of Mathematics expects the author's institution to pay page charges, and reserves the right to delay publication for nonpayment of charges in case of financial emergency.

100 reprints are provided free for each article, only if page charges have been substantially paid. Additional copies may be obtained at cost in multiples of 50 .

The Pacific Journal of Mathematics is issued monthly as of January 1966. Regular subscription rate: $\$ 72.00$ a year (6 Vols., 12 issues). Special rate: $\$ 36.00$ a year to individual members of supporting institutions.

Subscriptions, orders for back numbers, and changes of address should be sent to Pacific Journal of Mathematics, 103 Highland Boulevard, Berkeley, California, 94708.

PUBLISHED BY PACIFIC JOURNAL OF MATHEMATICS, A NON-PROFIT CORPORATION

Printed at Kokusai Bunken Insatsusha (International Academic Printing Co., Ltd.), 8-8, 3-chome, Takadanobaba, Shinjuku-ku, Tokyo 160, Japan. 


\section{Pacific Journal of Mathematics}

Patricia Andresen and Marvin David Marcus, Weyl's inequality and

quadratic forms on the Grassmannian .......................

George Bachman and Alan Sultan, Regular lattice measures: mappings and

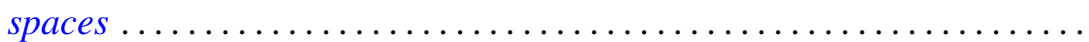

David Geoffrey Cantor, On certain algebraic integers and approximation by rational functions with integral coefficients ...................

James Richard Choike, On the value distribution of functions meromorphic in the unit disk with a spiral asymptotic value ..................

David Earl Dobbs, Divided rings and going-down................ 353

Mark Finkelstein and Robert James Whitley, Integrals of continuous

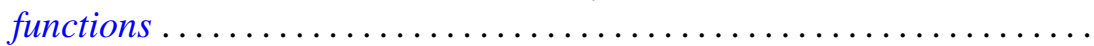

Ronald Owen Fulp and Joe Alton Marlin, Integrals of foliations on manifolds with a generalized symplectic structure ...............

Cheong Seng Hoo, Principal and induced fibrations .................

Wu-Chung Hsiang and Richard W. Sharpe, Parametrized surgery and

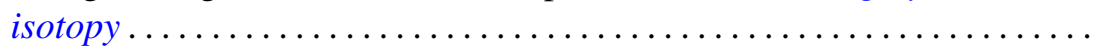

Surender Kumar Jain, Surjeet Singh and Robin Gregory Symonds, Rings whose proper cyclic modules are quasi-injective .................

Pushpa Juneja, On extreme points of the joint numerical range of commuting normal operators...

Athanassios G. Kartsatos, Nth order oscillations with middle terms of order $N-2$.

John Keith Luedeman, The generalized translational hull of a

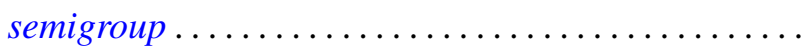

Louis Jackson Ratliff, Jr., The altitude formula and DVR's ...

Ralph Gordon Stanton, C. Sudler and Hugh C. Williams, An upper bound for the period of the simple continued fraction for $\sqrt{D}$...

David Westreich, Global analysis and periodic solutions of second order systems of nonlinear differential equations...

David Lee Armacost, Correction to: "Compactly cogenerated LCA

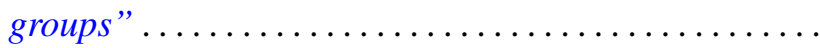

Jerry Malzan, Corrections to: "On groups with a single involution" .

David Westreich, Correction to: "Bifurcation of operator equations with unbounded linearized part" ...................... 